\title{
Umbilical cord blood versus unrelated donor transplantation in adults with primary refractory or relapsed acute myeloid leukemia: a report from Eurocord, the Acute Leukemia Working Party and the Cord Blood Committee of the Cellular Therapy and Immunobiology Working Party of the EBMT
}

Frédéric Baron (1) ', Myriam Labopin 2,3,4,5, Annalisa Ruggeri ${ }^{2,6,7}$, Gerhard Ehninger ${ }^{8}$, Fransesca Bonifazi (1) Matthias Stelljes ${ }^{10}$, Jaime Sanz ${ }^{11}$, Gernot Stuhler ${ }^{12}$, Alberto Bosi ${ }^{13}$, Nicolaus Kröger ${ }^{14}$, Maria Teresa Van Lint ${ }^{15}$, Arnold Ganser $^{16}$, Edouard Forcade ${ }^{17}$, Mohamad Mohty ${ }^{2,3,4,5}$, Eliane Gluckman ${ }^{6}$ and Arnon Nagler 3 ,18

\begin{abstract}
The role of umbilical cord blood transplantation (CBT) in acute myeloid leukemia (AML) patients with active disease at allogeneic hematopoietic cell transplantation (allo-HCT) remains poorly investigated. In this study, we compared transplantation outcomes of 2963 patients with primary refractory or relapsed AML given CBT, 10/10 HLA-matched UD, or 9/10 HLA-matched UD allo-HCT from 2004 to 2015 at EBMT-affiliated centers. Neutrophil engraftment and complete remission rates in CBT, UD 10/10, and UD 9/10 recipients were 75 and 48\%, 93 and 69\%, and 93 and 70\%, respectively. In multivariate Cox analyses, in comparison with CBT $(n=285)$, UD 10/10 recipients $(n=2001)$ had a lower incidence of relapse ( $H R=0.7, P=0.001)$, a lower incidence of non relapse mortality $(H R=0.6, P<0.001)$, better GVHD-free and leukemia-free survival (GRFS, $H R=0.8, P<0.001$ ) and better survival $(H R=0.6, P<0.001)$. Further, in comparison with CBT, 9/10 UD recipients $(n=677)$ also had a lower incidence of relapse (HR $=0.8, P=0.02)$, a lower incidence of nonrelapse mortality ( $H R=0.7, P=0.008)$, better GRFS (HR=0.8, $P=0.01)$ and better survival $(H R=0.7$, $P<0.001)$. In summary, these data suggest that in AML patients with active disease at transplantation, allo-HCT with UD results in better transplantation outcomes than CBT.
\end{abstract}

Correspondence: Frédéric Baron (f.baron@ulg.ac.be)

${ }^{1} \mathrm{GIGA}$ and CHU of Liege, University of Liege, Liege, Belgium

${ }^{2}$ Department of Haematology, Saint Antoine Hospital, Paris, France

Full list of author information is available at the end of the article.

These author's contributed equally: Frédéric Baron, Myriam Labopin, Annalisa

Ruggeri, Mohamad Mohty, Eliane Gluckman, Arnon Nagler.

\section{Introduction}

Allogeneic hematopoietic stem cell transplantation (allo-HCT) has remained the only potentially curative option for most patients with relapsed or primary refractory acute myeloid leukemia $(\mathrm{AML})^{1,2}$. This

\section{(c) The Author(s) 2019}

(c) Open Access This article is licensed under a Creative Commons Attribution 4.0 International License, which permits use, sharing, adaptation, distribution and reproduction BY in any medium or format, as long as you give appropriate credit to the original author(s) and the source, provide a link to the Creative Commons license, and indicate if changes were made. The images or other third party material in this article are included in the article's Creative Commons license, unless indicated otherwise in a credit line to the material. If material is not included in the article's Creative Commons license and your intended use is not permitted by statutory regulation or exceeds the permitted use, you will need to obtain permission directly from the copyright holder. To view a copy of this license, visit http://creativecommons.org/licenses/by/4.0/. 
approach is thought to rely mostly on graft-versusleukemia $(\mathrm{GvL})$ effects for tumor eradication ${ }^{3-5}$.

For adult patients with AML in complete remission (CR) who lack a suitable human leukocyte antigen (HLA)identical sibling, and cord blood transplantation (CBT) has proved to be an adequate alternative to HLA-matched unrelated (UD) bone marrow (BM)/peripheral blood stem cell (PBSC) transplantation ${ }^{6-8}$.

We recently compared GvL effects following lowintensity non myeloablative conditioning regimen according to donor type ${ }^{9}$. We observed that, in comparison with patients given grafts from HLA-matched unrelated donor (UD 10/10), those receiving CBT had similar overall survival (OS) but better GVHD-free and relapsefree survival (GRFS). These results are in line with another study from the Fred Hutchinson Cancer Research Center that demonstrated that, among AML patients in CR but with minimal residual disease at transplantation, those receiving CBT had at least as good overall survival (OS) than those given grafts from UD10/10, and better OS than those receiving grafts from HLA-mismatched UD ${ }^{10}$.

We hypothesized that, since CBT provides better outcome than UD in AML patients with minimal residual disease at transplantation, transplantation outcomes might be better with CBT than with UD in AML patients with active disease at transplantation. In order to challenge this hypothesis, we performed a large registry study comparing CBT with UD 10/10 or 1-antigen HLA-mismatched UD (UD 9/10) in patients with primary refractory or untreated/refractory relapsed AML.

\section{Patients and Methods \\ Data collection}

This is a retrospective, multicenter registry-based study performed by the Acute Leukemia Working Party (ALWP) of the European society for Blood and Marrow Transplantation (EBMT) and by Eurocord. EBMT registry is a voluntary working group of more than 500 transplant centers, participants of which are required once a year to report all consecutive stem cell transplantations and follow-up. Audits are routinely performed to determine the accuracy of the data. Eurocord collects data on CBT performed in $>50$ countries worldwide and $>500$ transplant centers, mainly EBMT centers.

Inclusion criteria were adult ( $\geq 18$ years) patients, de novo or secondary AML, primary refractory (defined as absence of CR ( $<5 \%$ marrow blasts) achievement after induction chemotherapy ${ }^{1}$ ) or in first or second relapse at transplantation, transplantation between 2004 and 2015, and either a 10/10 HLA-matched unrelated donor (UD 10/10), a 9/10 HLA-matched unrelated donor (UD 9/10), or a single or double CBT. For UD, HLA-A, HLA-B, HLA-C, HLA-DR, and HLA-DQ were typed at the allelic level. For CBT, HLA-compatibility requirements followed the current practice of antigen level typing for HLA-A and HLA-B and allele level typing of HLA-DRB1. CB units were 4-6/6 HLA-A, HLA-B, and HLA-DRB1 matched to the recipient and to the other unit in case of double CBT in most patients ${ }^{11,12}$. HLA disparities between each unit and the recipient and between the two units were not necessarily at the same loci. Grading of acute and chronic GVHD was performed using established criteria ${ }^{13}$.

For the purpose of this study, all necessary data were collected according to EBMT guidelines.

\section{Ethics approval and consent to participate}

The scientific boards of the ALWP of EBMT and of Eurocord approved this study. Since 1990, patients have provided informed consent authorizing the use of their personal information for research purposes.

\section{Statistical analyses}

Data from all patients meeting the inclusion/exclusion criteria were included in the analyses. Start time was date of transplant for all endpoints. Neutrophil engraftment was defined as first of 3 consecutive days with a neutrophil count of at least $0.5 \times 10^{9} / \mathrm{L}$.

Cumulative incidence functions were used for relapse incidence and non relapse mortality (NRM) in a competing risk setting, since death and relapse were competing together. For estimating the cumulative incidence of engraftment and chronic GVHD, death was considered as a competing event. Overall (OS) and leukemia-free (LFS) survivals were estimated using the Kaplan-Meier estimates. GVHD and relapse-free survival (GRFS) was defined as being alive with neither grade III-IV acute GVHD, extensive chronic GVHD nor disease relapse ${ }^{14}$.

The main characteristics at diagnosis and at transplantation were compared between CBT and $10 / 10$ or $9 / 10$ UD groups using Kruskall Walis tests for quantitative variables, Chi-square test or Fisher exact test for categorical variables. Univariate analyses were done using Gray's test for cumulative incidence function and log rank test for OS and LFS.

Associations between donor type and transplantation outcomes were evaluated in multivariable analyses, using Cox proportional hazards. We used propensity scores (PS) matching to control for pre-treatment imbalances on observed variables. The following factors were included in the propensity score model: age, year of transplant, status at transplantation (primary refractory or relapse), diagnosis (de novo or secondary AML), sex matching (female to male vs other), patient and donor CMV serology, conditioning intensity (RIC or MAC). The estimation of propensity score was performed using generalized boosted models ${ }^{15}$. We weighted the 3 groups receiving either CBT, $10 / 10$, or $9 / 10$ UD by estimating the Average Treatment Effect (ATE). We checked the balance between 
Table 1 Patient and transplant characteristics

\begin{tabular}{|c|c|c|c|c|}
\hline & $\begin{array}{l}\text { CBT } \\
(n=285)\end{array}$ & $\begin{array}{l}\text { UD } 10 / 10 \\
(n=2001)\end{array}$ & $\begin{array}{l}\text { UD 9/10 } \\
(n=677)\end{array}$ & $P$ value $^{\mathrm{a}}$ \\
\hline $\begin{array}{l}\text { Median patient age, year } \\
\text { (range) }\end{array}$ & $47(18-71)$ & $55(18-77)$ & $55(18-77)$ & $<0.001$ \\
\hline $\begin{array}{l}\text { Median follow-up (pts } \\
\text { alive), month (range) }\end{array}$ & $23(2-121)$ & $22(1-146)$ & $27(1-136)$ & $<0.001$ \\
\hline $\begin{array}{l}\text { Median year of } \\
\text { transplantation }\end{array}$ & 2009 & 2011 & 2011 & $<0.001$ \\
\hline Recipient gender M, \# (\%) & $139(49)$ & $1090(55)$ & $358(53)$ & 0.2 \\
\hline $\begin{array}{l}\text { F donor to M recipient, } \\
\text { \# (\%) }\end{array}$ & $61(24)$ & $226(12)$ & $113(17)$ & $<0.001$ \\
\hline \multicolumn{5}{|c|}{ Karnofsky performance status at Tx } \\
\hline$<80$ & $37(15)$ & $268(14)$ & $86(13)$ & 0.8 \\
\hline$>=80$ & $216(85)$ & $1611(86)$ & $555(87)$ & \\
\hline Missing & 32 & 122 & 36 & \\
\hline \multicolumn{5}{|l|}{ Diagnosis, \# (\%) } \\
\hline De novo AML & $204(72)$ & $1282(64)$ & $435(64)$ & 0.04 \\
\hline Secondary AML & $81(28)$ & $719(36)$ & $242(36)$ & \\
\hline \multicolumn{5}{|c|}{ Status at transplantation, \# (\%) } \\
\hline Primary refractory & $106(37)$ & $999(50)$ & $286(42)$ & $<0.001$ \\
\hline First relapse & $146(50)$ & $876(44)$ & $336(50)$ & \\
\hline Second relapse & $37(13)$ & $126(6)$ & $55(8)$ & \\
\hline \multicolumn{5}{|l|}{ Cytogenetics, \# (\%) } \\
\hline Good risk ${ }^{b}$ & $8(4)$ & $56(5)$ & $23(5)$ & 0.001 \\
\hline Intermediate risk ${ }^{c}$ & $44(25)$ & $178(15)$ & $75(18)$ & \\
\hline High risk ${ }^{d}$ & $46(26)$ & $201(17)$ & $85(20)$ & \\
\hline Secondary AML & $81(45)$ & $719(62)$ & $242(57)$ & \\
\hline Not reported/failed & 106 & 847 & 252 & \\
\hline \multicolumn{5}{|l|}{ FLT3-ITD, \# (\%) } \\
\hline Negative & $28(58)$ & $150(61)$ & $52(52)$ & 0.3 \\
\hline Positive & $20(42)$ & $94(39)$ & $48(48)$ & \\
\hline Missing & 237 & 1757 & 577 & \\
\hline \multicolumn{5}{|l|}{ Stem cell source } \\
\hline Bone marrow & $152(8)$ & $56(8)$ & & \\
\hline $\begin{array}{l}\text { Peripheral blood stem } \\
\text { cells }\end{array}$ & $1849(92)$ & $620(92)$ & & \\
\hline Single CBT & & & $175(61)$ & \\
\hline Double CBT & & & $110(39)$ & \\
\hline $\begin{array}{l}\text { Patient CMV seropositive, } \\
\text { \# (\%) }\end{array}$ & $169(69)$ & $1222(63)$ & $430(66)$ & 0.1 \\
\hline \multicolumn{5}{|l|}{ Conditioning intensity, \# (\%) } \\
\hline Myeloablative (MAC) & $148(52)$ & $901(46)$ & $276(42)$ & 0.009 \\
\hline Reduced-intensity (RIC) & $135(48)$ & $1062(54)$ & $387(58)$ & \\
\hline \multicolumn{5}{|l|}{ Conditioning regimen, \# (\%) } \\
\hline $\mathrm{Cy}-\mathrm{TBI}$ & $106(39)$ & $208(11)$ & $70(11)$ & $<0.001$ \\
\hline Flu-TB| & $10(4)$ & $204(10)$ & $44(7)$ & \\
\hline BuCy & $13(5)$ & $207(11)$ & $60(9)$ & \\
\hline BuFlu & $6(2)$ & $323(17)$ & $98(15)$ & \\
\hline FluMel & $15(5)$ & $255(13)$ & $98(15)$ & \\
\hline TBF & $66(24)$ & $32(2)$ & $24(4)$ & \\
\hline Flamsa TBI/chemo & $23(8)$ & $454(23)$ & $184(28)$ & \\
\hline Other & $35(13)$ & $273(14)$ & $83(12)$ & \\
\hline Missing & 11 & 45 & 16 & \\
\hline \multicolumn{5}{|l|}{ In vivo T-cell depletion, \# (\%) } \\
\hline Yes & $98(38)$ & $485(25)$ & $101(15)$ & $<0.001$ \\
\hline No & $159(62)$ & $1491(75)$ & $569(85)$ & \\
\hline Missing & 28 & 25 & 7 & \\
\hline
\end{tabular}

Table 1 continued

\begin{tabular}{lllll}
\hline & $\begin{array}{l}\text { CBT } \\
(\boldsymbol{n}=\mathbf{2 8 5})\end{array}$ & $\begin{array}{l}\text { UD 10/10 } \\
(\boldsymbol{n}=\mathbf{2 0 0 1 )})\end{array}$ & $\begin{array}{l}\text { UD 9/10 } \\
(\boldsymbol{n}=\mathbf{6 7 7})\end{array}$ & P value $^{\mathbf{a}}$ \\
\hline $\begin{array}{l}\text { Postgrafting immunosuppression, \# (\%) } \\
\text { CSP alone }\end{array}$ & $58(23)$ & $206(11)$ & $64(10)$ & $<0.001$ \\
$\begin{array}{l}\text { CSP (or tacro) + MTX } \\
+/- \text { MMF }\end{array}$ & $13(5)$ & $686(35)$ & $220(33)$ & \\
$\begin{array}{l}\text { CSP (or tacro) + MMF } \\
\text { Post-transplant }\end{array}$ & $174(69)$ & $966(50)$ & $345(52)$ & \\
$\begin{array}{l}\text { cyclophosphamide } \\
\text { Other }\end{array}$ & $7(3)$ & $28(1)$ & $13(2)$ & \\
Missing & $1(0)$ & $61(3)$ & $24(4)$ & \\
\hline
\end{tabular}

$M$ male, $C R$ complete remission, \# number of patients, UD unrelated donor, $C B T$ cord blood transplantation, CSP cyclosporine, MMF mycophenolate mofetil, FLT3-ITD FMS-related tyrosine kinase 3 internal tandem duplication, Cy cyclophosphamide, TBI total body irradiation, Bu busulfan, Flu fludarabine, Mel Melphalan, TBF thiotepa + busulfan + fludarabine, Flamsa fludarabine + amsacrine + cytarabine, MTX methotrexate

${ }^{\mathrm{a}}$ calculated with $X^{\mathrm{b}}$ statistics for categorical variables and Mann-Whitney test for continuous variables

${ }^{b}$ defined as $\mathrm{t}(8 ; 21), \mathrm{t}(15 ; 17)$, inv or del $(16)$, or acute promyelocyticleukemia these abnormalities only or combined with others

'defined as all cytogenetics not belonging to the good or high risk (including trisomias)

ddefined as 11q23 abnormalities, complex caryotype, abnormalities of chromosomes 5 and 7

the groups looking to ATE Weighted means. Then, we used pairwise ATEs to fit weighted KM and Cox models. All tests were two sided. The type I error rate was fixed at 0.05 for determination of factors associated with time to event outcomes. Analyses were performed using the $\mathrm{R}$ statistical software version 3.4.0. Propensity score analysis was performed using the mnps function of the Twang package (http://cran.rproject.org/web/packages/twang/ vignettes/twang.pdf), and weighted analyses using the survey package.

\section{Results}

Patients and donors

Data from 2963 patients with primary or secondary AML were included in this study (Table 1$)$. Two hundred eighty five patients underwent a single $(n=175)$ or double $(n=$ 110) CBT, 2001 an UD $10 / 10$ and 677 an UD 9/10 alloHCT. In comparison to $10 / 10$ UD or 9/10 UD recipients, CBT patients were younger (48 vs. 55 and 55 years, respectively, $P<0.001$ ), were less frequently transplanted with primary refractory disease (37\% vs. 50 and $42 \%$, respectively, $P=0.009$ ), and were more frequently conditioned with a myeloablative regimen (52\% vs. 46 and $42 \%$, respectively, $P<0.001)$. However, they received less frequently in vivo $\mathrm{T}$ cell depleting agents such as ATG or alemtuzumab ( $62 \%$ vs. 75 and $85 \%$, respectively, $P<0.0001$ ).

\section{Engraftment and GVHD}

Cumulative incidence of neutrophil engraftment at day 60 was $73 \%, 94$ and $93 \%$ in CBT, UD 10/10 and UD 9/10, respectively $(P<0.001)$. Cumulative incidence of grades 
II-IV acute GVHD were $27 \%, 30$ and $36 \%$ in CBT, UD10/ 10 , and UD $9 / 10$ recipients, respectively $(P=0.002)$. For grade III-IV acute GVHD, the figures were $12 \%, 13$ and $17 \%$, respectively $(P=0.02)$. Further, the 2 -year cumulative incidences of chronic and extensive chronic GVHD were lower in CBT patients (16 and 6\%, respectively) than in UD $10 / 10$ (28 and $12 \%$, respectively) or UD $9 / 10$ patients $(29$ and $14 \%$, respectively) $\quad(P<0.001$ and $P=0.004$, respectively).

\section{CR achievement, relapse and NRM}

Following transplantation, $69 \%$ of UD 10/10 recipients, $70 \%$ of $9 / 10$ recipients versus $48 \%$ of $\mathrm{CBT}$ recipients achieved a $C R$ within 100 days $(P<0.001)$. Two-year incidences of relapse and NRM were 43 and 26\% in UD 10/10 recipients, 44 and 33\% in UD 9/10 recipients and 47 and $38 \%$ in CBT recipients, respectively (Fig. 1). In multivariate Cox analyses, in comparison to CBT patients, UD $10 / 10$ recipients had a lower incidence of relapse (HR $=0.7, P=0.001)$ and a lower incidence of NRM $(\mathrm{HR}=0.6, P<0.001)$. Further, in comparison with CBT patients UD 9/10 recipients had also a lower incidence of relapse $(H R=0.8, P=0.02)$ and a lower incidence of NRM $(\mathrm{HR}=0.7, P=0.008)$.

Other factors associated with the relapse incidence in multivariate analysis included secondary versus primary AML $(\mathrm{HR}=0.8, \quad P=0.01)$, relapsed versus primary refractory AML $(\mathrm{HR}=1.2, P=0.02)$ and older age at transplantation (HR per ten year $=0.9, P<0.001$ ). Further, other factors associated with NRM in multivariate analyses included older age $(\mathrm{HR}=1.1, P<0.001)$, female donor to male recipient $(\mathrm{HR}=1.2, P=0.05)$ and patient CMV seropositivity $(\mathrm{HR}=1.3, P=0.002)$.

\section{OS, LFS, GRFS}

At 2-year, OS, LFS, and GRFS were 37,31 , and $20 \%$ in UD $10 / 10$ recipients, 27,23 , and $16 \%$ in UD $9 / 10$ recipients, and 18, 16 and $11 \%$ in CBT patients, respectively. In multivariate Cox analyses, in comparison with CBT, UD 10/10 recipients had a better LFS (HR $=0.6, P<$ $0.001)$, a better GRFS (HR $=0.8, P<0.001)$ and a better OS (HR $=0.6, P<0.001)$ (Table 2). Further, in comparison to CBT patients, UD 9/10 recipients had also a better LFS (HR $=0.7, P<0.001$ ), a better GRFS (HR $=0.8, P=$ $0.01)$ and a better OS $(\mathrm{HR}=0.7, P<0.001)($ Table 2$)$.

Other factors associated with transplantation outcomes in multivariate analyses included relapsed versus primary refractory AML that predicted poor LFS $(\mathrm{HR}=1.1, P=$ $0.02)$ and $\mathrm{OS}(\mathrm{HR}=1.1, P=0.04)$, while patient $\mathrm{CMV}$ seropositivity predicted for poor LFS $(\mathrm{HR}=1.2, P=$ $0.003)$, OS (HR $=1.2, P=0.003)$, and GRFS $(\mathrm{HR}=1.1$, $P=0.01)$.

Among CBT recipients, 38\% of patients died because of the original disease, $24 \%$ because of an infection, and $5 \%$ because of GVHD. Among UD 10/10 recipients the figures were 31,13 , and $7 \%$ respectively. Finally, among 9/10 recipients, the figures were 34,15 , and $13 \%$, respectively.

\section{Propensity score analysis}

Given the differences in the study population between the 3 groups we also performed analyses weighted with propensity score. These analyses showed that in comparison with CBT recipients, UD 10/10 patients had lower incidences of relapse and of nonrelapse mortality translating to better LFS, OS, and GRFS (Table 3). Further, in comparison with CBT recipients, UD 9/10 patients had a lower incidence of relapse, a not significantly different incidence of NRM, as well as better LFS, OS, and GRFS (Table 3).

\section{Discussion}

Based on recent data demonstrating that in patients with MRD at transplantation, CBT did as least as good as UD transplantation, we performed a survey comparing the outcomes of patients with refractory/relapsed AML transplanted with a UD vs. a CB; several observations were made.

First, we observed a high incidence of primary graft failure, as well as a low incidence of CR achievement in CBT recipients. The high incidence of graft failure in CBT recipient was due in a large part to early AML progression precluding neutrophil engraftment since only $49 \%$ of CBT recipients achieved a $C R$ after transplantation. This suggests that the kinetic of GvL effects is slower in CBT than in UD recipients. This might be due to the lower number of $\mathrm{T}$ cells infused with CBT, as well as to their mostly immature status ${ }^{16}$.

Second, we observed a significantly higher NRM in CBT patients than in UD recipients. While this could probably be attributed in a part to the slow neutrophil engraftment associated with CBT, it could also be due to delayed immune reconstitution in CBT patients since the higher non relapse mortality in CBT than in UD recipients was due to infections. One cannot include that the large use of ATG among CBT recipients was in part the cause of this high infection-related mortality among CBT recipients ${ }^{17}$. Recent advances in the field of CBT engineering are likely to improve the safety of CBT in AML patients with active disease at transplantation ${ }^{18-20}$.

Consequently to the high disease-related and infectionrelated mortality among CBT recipients, OS, LFS, and GRFS were significantly better both in UD $10 / 10$ and in UD 9/10 recipients than in CBT patients. These results are in contrast with those observed in AML patients in CR with or without MRD, where CBT did at least as good as UD allo-HCT ${ }^{8-10}$.

There are some limitations in our study. They include the heterogeneity in patients characteristics between the 


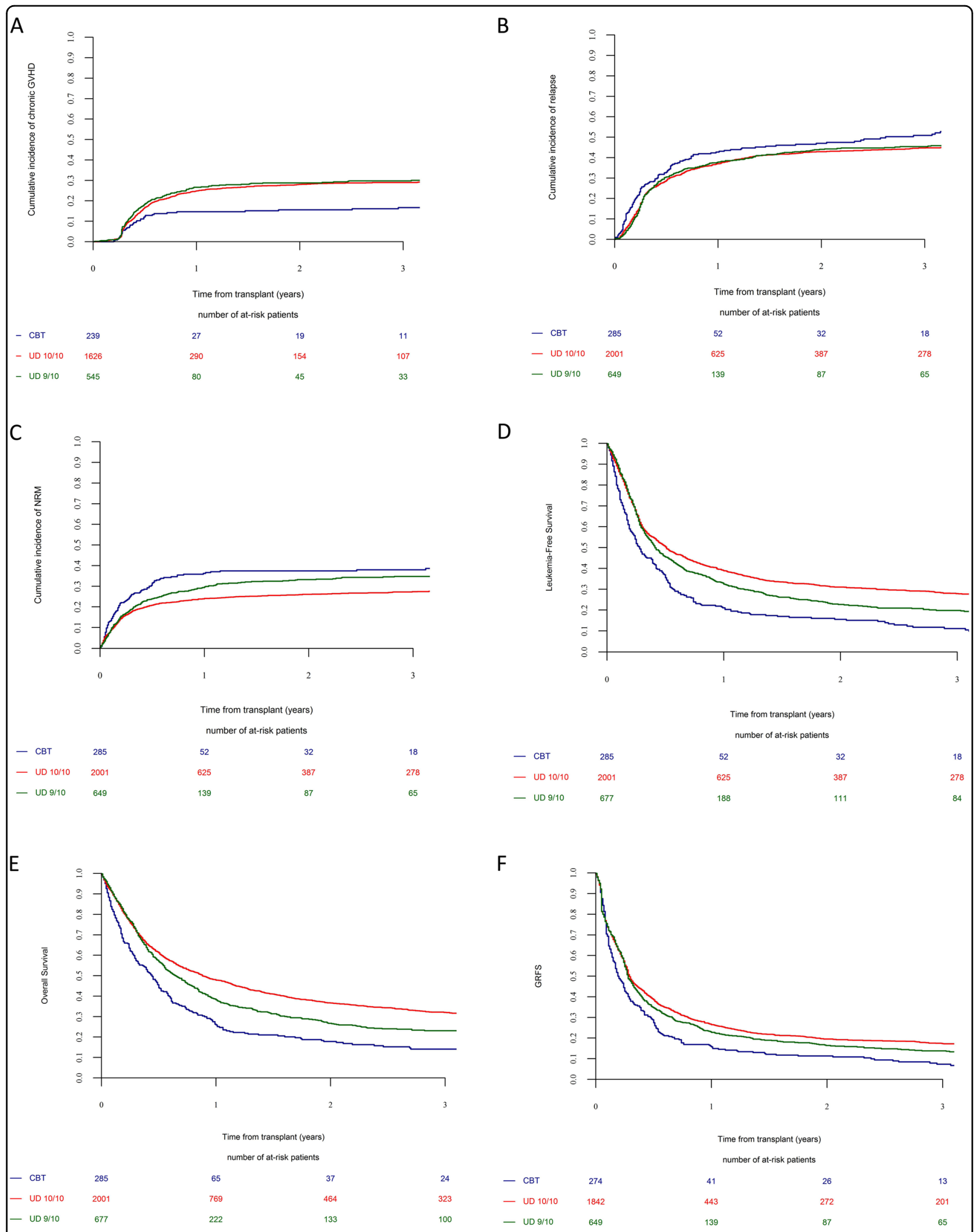

Fig. 1 Impact of donor type on transplantation outcomes. a Chronic GVHD. b Relapse. c Non relapse mortality. $\mathbf{d}$ LFS. e OS. $\mathbf{f}$ GRFS 
Table 2 Impact of donor types on transplantation outcomes in multivariate Cox models

\begin{tabular}{lll}
\hline HR & $95 \% \mathrm{Cl}$ & $P$ \\
\hline
\end{tabular}

Relapse

CBT

UD 10/10

UD $9 / 10$

1

0.7

0.8

$0.6-0.9$

$0.6-1.0$

$<0.001$

0.02

Non relapse mortality

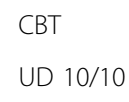

UD $9 / 10$

\section{1}

0.6

0.7

$0.5-0.9$

$<0.001$

0.007

Leukemia-free survival

$$
\text { CBT }
$$

UD 10/10

\section{1}

UD $9 / 10$

$0.6-0.8$

$<0.001$

0.7

$0.6-0.9$

$<0.001$

Overall survival

$$
\text { CBT }
$$

UD 10/10

$$
1
$$

GVHD-free and relapse-free survival

\begin{tabular}{llll} 
CBT & 1 & - & - \\
UD 10/10 & 0.8 & $0.7-0.9$ & $<0.001$ \\
UD 9/10 & 0.8 & $0.7-1.0$ & 0.01 \\
\hline
\end{tabular}

different groups and the lack of relevant data in the database such as blast counts at transplantation, comorbidity score, or donor $\mathrm{T}$ cell reconstitution after alloHCT. We tried to address the heterogeneity question by comparing transplantation outcomes between the three different groups by using both multivariate Cox models and propensity weighted Cox models.

In summary, these data suggest that in AML patients with active disease at transplantation, allo-HCT with UD results in better transplantation outcomes than CBT.

\section{List of institutions}

The EBMT registry is a voluntary working group of more than 500 transplant centers, participants of which are required once a year to report all consecutive stem cell transplantations and follow-up. The list of institutions reporting data included in this study is provided in the supplemental data.

\section{Acknowledgements}

We thank Emmanuelle Polge and Audrey Mailhol from the office of the ALWP of EBMT, Chantal Kenzey and Fernanda Volt from the Eurocord study office. F.B. is Senior Research Associate at the National Fund for Scientific Research (FNRS) Belgium.

Table 3 Impact of donor types on transplantation outcomes using Cox models weighted on propensity score

\begin{tabular}{llll}
\hline & HR & $\mathbf{9 5 \% ~ C l}$ & $\boldsymbol{P}$ \\
\hline Relapse & & & \\
CBT & 1 & - & - \\
UD 10/10 & 0.7 & $0.5-0.8$ & $<0.001$ \\
UD 9/10 & 0.87 & $0.5-0.9$ & 0.005 \\
Non relapse mortality & & & \\
CBT & 1 & - & - \\
UD 10/10 & 0.6 & $0.5-0.8$ & $<0.001$ \\
UD 9/10 & 0.8 & $0.6-1.1$ & 0.1 \\
Leukemia-free survival & & & - \\
CBT & 1 & - & $<0.001$ \\
UD 10/10 & 0.6 & $0.5-0.8$ & 0.002
\end{tabular}

\section{Overall survival}

CBT
UD $10 / 10$

$0.6-0.9$

0.002

UD $9 / 10$

0.6

0.8

GVHD-free and relapse-free survival

$$
\begin{aligned}
& \text { CBT } \\
& \text { UD } 10 / 10 \\
& \text { UD } 9 / 10
\end{aligned}
$$

1
0.7
0.8

\section{$0.5-0.8$}

$<0.001$

$0.6-0.9$

0.004

\section{Author details}

${ }^{1}$ GIGA and CHU of Liege, University of Liege, Liege, Belgium. ${ }^{2}$ Department of Haematology, Saint Antoine Hospital, Paris, France. ${ }^{3}$ EBMT Paris Study Office/ CEREST-TC, Paris, France. ${ }^{4}$ INSERM UMR 938, Paris, France. ${ }^{5}$ Sorbonne University, Paris, France. ${ }^{6}$ Eurocord, Saint Louis Hospital, Paris, France. ${ }^{7}$ Dipartimento di Oncoematologia e terapie cellulari, Ospedale Pediatrico Bambino Gesù, Roma, Italy. ${ }^{8}$ Universitaetsklinikum Dresden, Medizinische Klinik und Poliklinik I,

Dresden, Germany. 'S.Orsola-Malpighi Hospital, Institute of Hematology and Medical Oncology, Bologna University, Bologna, Italy. ${ }^{10}$ Department of Hematology/Oncology, University of Münster, Münster, Germany.

${ }^{11}$ Hematology Department, University Hospital La Fe, Valencia, Spain.

${ }^{12}$ Deutsche Klinik für Diagnostik, KMT Zentrum, Wiesbaden, Germany.

${ }^{13}$ Department of Hematology, Azienda Ospedaliera Universitaria Careggi, Firenze, Italy. ${ }^{14}$ Bone Marrow Transplantation Centre, University Hospital Eppendorf, Hamburg, Germany. ${ }^{15}$ Department of Haematology II, Ospedale San Martino, Genova, Italy. ${ }^{16}$ Department of Hematology, Hemostasis, Oncology, and Stem Cell Transplantation, Hannover Medical School, Hannover, Germany. ${ }^{17} \mathrm{CHU}$ Bordeaux, Service d'hematologie et therapie cellulaire, F33000 Bordeaux, France. ${ }^{18}$ Division of Hematology and Bone Marrow Transplantation, The Chaim Sheba Medical Center, Tel-Hashomer, Ramat-Gan, Israel

\section{Author contributions}

F.Ba. wrote the manuscript, designed the study and interpreted the data; M.L. designed the study, analyzed and interpreted the data, and edited the manuscript; A.R., E.G., and A.N. designed the study, interpreted the data and edited the manuscript; M.M. helped in the study design and edited the manuscript; all other authors reviewed the manuscript and provided clinical data. All authors approved the final version of the manuscript. 


\section{Conflict of interest}

The authors declare that they have no conflict of interest.

\section{Publisher's note}

Springer Nature remains neutral with regard to jurisdictional claims in published maps and institutional affiliations.

\section{Received: 29 July 2018 Accepted: 20 March 2019}

Published online: 12 April 2019

\section{References}

1. Brissot, E. et al. Comparison of matched sibling donors versus unrelated donors in allogeneic stem cell transplantation for primary refractory acute myeloid leukemia: a study on behalf of the Acute Leukemia Working Party of the EBMT. J. Hematol. Oncol. 10(1), 130 (2017).

2. Gyurkocza, B., Lazarus, H. M. \& Giralt, S. Allogeneic hematopoietic cell transplantation in patients with AML not achieving remission: potentially curative therapy. Bone Marrow Transplant. 52(8), 1083-1090 (2017).

3. Baron, F. et al. Impact of graft-versus-host disease after reduced-intensity conditioning allogeneic stem cell transplantation for acute myeloid leukemia: a report from the Acute Leukemia Working Party of the European group for blood and marrow transplantation. Leukemia 26(12), 2462-2468 (2012).

4. Baron, F. et al. Occurrence of graft-versus-host disease increases mortality after umbilical cord blood transplantation for acute myeloid leukemia: a report from Eurocord and the ALWP of the EBMT. J. Intern. Med. 283(2), 178-189 (2018).

5. Dickinson, A. M. et al. Graft-versus-leukemia effect following hematopoietic stem cell transplantation for leukemia. Front. Immunol. 8, 496 (2017).

6. Weisdorf, D. et al. Alternative donor transplantation for older patients with acute myeloid leukemia in first complete remission: a center for international blood and marrow transplant research-eurocord analysis. Biol. Blood Marrow Transplant. 20(6), 816-822 (2014).

7. Ruggeri, A. et al. Comparison of outcomes after single or double cord blood transplantation in adults with acute leukemia using different types of myeloablative conditioning regimen, a retrospective study on behalf of Eurocord and the Acute Leukemia Working Party of EBMT. Leukemia 28(4), 779-786 (2014).
8. Eapen, M. et al. Effect of graft source on unrelated donor haemopoietic stemcell transplantation in adults with acute leukaemia: a retrospective analysis. Lancet Oncol. 11(7), 653-660 (2010).

9. Baron, F. et al. Impact of donor type in patients with AML given allogeneic hematopoietic cell transplantation after low-dose TBI-based regimen. Clin. Cancer Res. 24(12), 2794-2803. https://doi.org/10.1158/1078-0432.CCR-17-3622 (2018).

10. Milano, F. et al. Cord-blood transplantation in patients with minimal residual disease. N. Engl. J. Med. 375(10), 944-953 (2016).

11. Baron, F. et al. Single- or double-unit UCBT following RIC in adults with AL: a report from Eurocord, the ALWP and the CTIWP of the EBMT. J. Hematol. Oncol. 10(1), 128 (2017).

12. Baron, F. et al. RIC versus MAC UCBT in adults with AML: a report from Eurocord, the ALWP and the CTIWP of the EBMT. Oncotarget $7(28)$, 43027-43038 (2016).

13. Glucksberg, $\mathrm{H}$. et al. Clinical manifestations of graft-versus-host disease in human recipients of marrow from HL-A-matched sibling donors. Transplantation 18, 295-304 (1974).

14. Ruggeri, A., Labopin, M., Ciceri, F., Mohty, M. \& Nagler, A. Definition of GVHDfree, relapse-free survival for registry-based studies: an ALWP-EBMT analysis on patients with AML in remission. Bone Marrow Transplant. 51(4), 610-611 (2016).

15. McCaffrey, D. F. et al. A tutorial on propensity score estimation for multiple treatments using generalized boosted models. Stat. Med. 32(19), 3388-3414 (2013).

16. Hiwarkar, P. et al. Cord blood transplantation recapitulates fetal ontogeny with a distinct molecular signature that supports CD4(+) T-cell reconstitution. Blood Adv. 1(24), 2206-2216 (2017).

17. Admiraal, R. et al. Excellent T-cell reconstitution and survival depend on low ATG exposure after pediatric cord blood transplantation. Blood 128(23), 2734-2741 (2016).

18. Delaney, C., Bollard, C. M. \& Shpall, E. J. Cord blood graft engineering. Biol. Blood Marrow Transplant. 19(1 Suppl), S74-78 (2013).

19. Baron, F., Ruggeri, A. \& Nagler, A. Methods of ex vivo expansion of human cord blood cells: challenges, successes and clinical implications. Exp. Rev. Hematol 9(3), 297-314. https://doi.org/10.1586/17474086.2016.1128321 (2016).

20. Wagner, J. E. Jr. et al. Phase I/II trial of stem Regenin-1 expanded umbilical cord blood hematopoietic stem cells supports testing as a stand-alone graft. Cell Stem Cell 18(1), 144-155 (2016). 